\title{
What Is the Evidence for Paediatric/Adolescent Bariatric Surgery?
}

\author{
Natalie Durkin $^{1}$ - Ashish P. Desai ${ }^{1}$ \\ Published online: 16 August 2017 \\ (C) The Author(s) 2017. This article is an open access publication
}

\begin{abstract}
Purpose of Review In spite of the increasing prevalence of severe and complex obesity in children, surgery as a potential management option is still not widely accepted. The purpose of this review is to examine the evidence for surgical options in the severely obese paediatric population. Increasing evidence supports early rather than later use of bariatric surgery in the treatment of extreme obesity.

Recent Findings Prior to 2007, the feasibility and safety of surgery have been reported by predominantly small, sporadic single-centre retrospective case series. Increasing long-term data is now emerging due to the formation of multi-centre prospective national consortiums with two large, prospective long-term outcome studies published within the last year aiding our understanding of the efficacy and safety of bariatric surgery within the adolescent population.

Summary It is increasingly clear that adolescent bariatric surgery outcomes are comparable to adults, with similar sustainable weight loss, resolution of co-morbidities and complication rates. However, these studies are solely from dedicated specialist adolescent centres and results may not be reproducible if not performed in regulated environments with specialist multi-disciplinary teams.
\end{abstract}

Keywords Paediatric $\cdot$ Bariatric surgery $\cdot$ Adolescent . Benefits $\cdot$ Complications $\cdot$ Sleeve gastrectomy

This article is part of the Topical Collection on Health Services and Programs

Ashish P. Desai

ashishdesai@nhs.net

1 Department of Paediatric Surgery, King's College Hospital, Denmark Hill, London SE9 5RS, UK

\section{Introduction}

Obesity is a global epidemic. Childhood obesity in particular has become an increasing concern due to the rising prevalence of associated co-morbidities including metabolic syndrome and diabetes, obstructive sleep apnoea syndrome (OSAS) and psychosocial impairments, at ever-younger ages. These co-morbidities have a cumulative health impact, making the duration of obesity increasingly important and predisposing patients to a significant risk of premature morbidity and mortality [1]. As such, childhood obesity is fast becoming the most significant threat to the health of our younger generations.

Lifestyle interventions have been shown to have limited success in treatment of severe obesity in children. In adults, sustained BMI reduction and significant and definitive risk reductions for developing cardiovascular, cancer, endocrine, infectious and psychiatric disorders have been found in surgical cohorts as compared to controls [2]. With such lifechanging results in the adult population, why not, then, apply the same intervention prior to the development of complications of co-morbidities in the paediatric population? This article aims to explain the rationale for paediatric bariatric surgery, review the growing evidence base of outcomes and explore the controversies that still exist in order to delineate the future of this growing problem.

\section{Prevalence of Childhood Obesity}

Defining obesity in children presents slightly different challenges to that in adults. The body mass index (BMI) provides a relatively good gauge of body fat, whilst being easy to measure and subsequently calculate. As such, standard levels of BMI classify obesity in adults; if the BMI $>30 \mathrm{~kg} / \mathrm{m}^{2}$, this is 
considered obese. Children have varying proportions of body fat at varying ages which also differs depending on gender. As such, fixed levels of BMI provide inaccurate comparisons.

In children, gender and age-specific BMI growth charts of large reference populations consider the pattern of growth over time. BMI Z-scores/standard deviation score (BMI-SDS), a measure of how many standard deviations a child's BMI is above the mean, are used to provide set definitions of obesity. The most widely used definition of 'severe' obesity is a BMI > 99th centile, broadly equivalent to a BMI Z-score of +2.5 , an adult BMI equivalent $30 \mathrm{~kg} / \mathrm{m}^{2}$ [3]. Adolescent BMI $>$ 99th centile has specifically been shown to have a strong positive correlation with adverse cardiovascular risk profile [1].

Prevalence of childhood obesity has risen by $47.1 \%$ between 1980 and 2013. Although the overall proportion of childhood obesity appears to have plateaued out over the last decade, there is no convincing evidence of a sustainable decline. The National Child Measurement Programme (NCMP) in the UK identified $21.9 \%$ children aged $4-5$ were classified as overweight or obese, a figure, which rose to one third of 10-11-year-olds [4]. Based on a definition of a BMI > 99th centile, $2.9 \%$ of girls and $3.9 \%$ of boys in the UK have severe obesity [5]. This finding is paralleled in other developed countries with the prevalence of severe obesity affecting approximately $4 \%$ of US adolescents [6]. This is an effect now seen in developing countries with a $60 \%$ increase in prevalence in recent years [7]. Childhood obesity is, therefore, a truly global phenomenon of growing concern.

\section{Effect of Lifestyle Intervention as Treatment Modality}

Community-based interventions have yet to demonstrate significant and sustainable results. A 2009 Cochrane review of combined behavioural and lifestyle management of paediatric obesity in 5230 patients as compared to controls demonstrated a -0.06 overall reduction of BMI-SDS in the under 12-year-olds, with only a slight improvement of -0.47 BMI-SDS in a more recent systematic review in $2014[8,9]$. A decrease in BMI-SDS of $<$ -0.25 was found in $<10 \%$ of the group. In addition, children with a lower BMI (30-35) achieved better results than those deemed to more extreme obesity (BMI 35+), indicating lifestyle intervention is more effective in those with lower BMI scores.

\section{Rationale for Surgery}

As discussed, lifestyle modification alone does not appear to achieve substantial and sustainable weight loss in obese adolescents. As far back as 1993, Must et al. studied the long term relationship between overweight and obese adolescents and cardiovascular morbidity and found increased mortality with coronary heart disease in men [10]. Similar increases in all-cause mortality have since been demonstrated in another study of 1.46 million white adults [11]. Failure of lifestyle management, increased mortality for untreated patients and early results of adolescent surgery have all indicated that adolescent bariatric surgery is a successful therapeutic option if lifestyle modifications fail.

\section{Eligibility Criteria}

There are multiple guidelines regarding criteria for surgery. Aikenhead et al. reviewed current guidelines worldwide and suggested that although they were all similar, they lacked uniformity regarding the age and severity of obesity at which intervention should be offered [12]. NICE (National Institute for Health and Clinical Excellence, UK) suggests that the surgery should only be carried out in this age group under exceptional circumstances, however, recommends following the same BMI criteria as adults [13]. The international paediatric endosurgery group (IPEG) recommends surgery in adolescents who have attained or almost attained adult stature with specific guidance (see Table 1) [14]. The unifying factor

Table 1 Adolescents being considered for bariatric surgery should fulfil all of the below criteria

$\checkmark$ Be very severely obese (BMI $\geq 40)$ with serious obesity related co-morbidities

$\checkmark$ Have attained or depending on the severity of co-morbidity, nearly attained adult stature

$\checkmark$ Have failed at least 6 months of organised conventional attempts at weight management

$\checkmark$ Demonstrated commitment to comprehensive paediatric psychological evaluation both before and after surgery and agree to avoid pregnancy for at least 1 year post-operatively

$\checkmark$ Be capable of and willing to adhere to nutritional guidelines post-operatively

$\checkmark$ Have decisional capacity and provide informed assent for surgical management.

Comorbid conditions

- Serious comorbidities

- Type 2 diabetes mellitus

- Obstructive sleep apnoea

- Pseudotumor cerebri

Less serious comorbidities

- Weight related arthropathy

- Hypertension

- Dyslipidaemia

- Venous stasis disease

- Panniculitis

- Urinary incontinence

- Significant impairment in activity of daily living

- Non-alcoholic fatty liver disease (includes steatohepatitis)

- Gastroesophageal reflux

- Severe psychosocial distress

Available at https://www.ipeg.org/morbidobesity/. Copyright (CInternational Paediatric Endosurgery Group (IPEG), used with permission 
in all guidelines appears to be recommending the use of multidisciplinary team (MDT) and the need for long-term followup.

There is no specific evidence for the use of a multidisciplinary team either for adults or adolescents undergoing bariatric surgery. Considering the complex nature of intervention, however, particularly at the vulnerable age of these patients, the MDT has become well established as the gold standard of care necessary to provide a safe and efficient service.

The team members recommended by the American Society of Metabolic and Obesity Surgeons (ASMBS) in 2012 [15] includes the following:

1. An experienced bariatric surgeon

2. A paediatric specialist; either a paediatrician with a specialty in endocrinology, gastroenterology, nutrition, and/ or adolescence or an internist or family practitioner with training in adolescent medicine.

3. A registered dietician with experience in treating obesity and working with children and families.

4. A mental health specialist; a psychiatrist or psychologist with specialty training in paediatrics $+/-$ adolescents and particular experience in treating eating disorders and obesity. In addition, the practitioner should have experience evaluating patients and families for bariatric surgery.

Further recommendations, although not essential, include a coordinator and an exercise physiologist or physical therapist to provide safe physical activity prescriptions to morbidly obese adolescents. Teams should follow up patients for at least for 2 years after surgery. Transition arrangements should be finalised with adult teams however as far as the authors are aware, there are no published guidelines about this.

\section{Outcomes}

\section{Weight Loss}

Evidence within the adult population demonstrates clear justification for bariatric surgery with regard to sustainable weight loss and improvement of co-morbidities. Chang et al. published a systematic review and meta-analysis of outcomes in 161,756 adult patients undergoing bariatric surgical procedures in 2014 . Both Roux-en-Y gastric bypass (RYGB) and laparoscopic sleeve gastrectomy (LSG) were shown to be effective in achieving weight loss with BMI loss of -15.9 and $-16.1 \mathrm{~kg} / \mathrm{m}^{2}$, respectively, at 5 years. The complication rate was $17 \%$ with a $7 \%$ reoperation rate and a $0.08 \%<30$-day mortality. Obesityrelated co-morbidities were found to resolve almost universally with remission of diabetes (92\%), hypertension (75\%), dyslipidaemia (76\%) and OSA (96\%) [16].
Despite such a wealth of adult evidence, there has been very little in the way of comparable studies from the adolescent population. Black et al. published a meta-analysis of adolescent bariatric surgical articles including 637 patients from 23 studies. The mean BMI change was $-13.5 \mathrm{~kg} / \mathrm{m}^{2}$ for combined procedures (RYGB, LSG, LAGB) with the largest drop in the RYGB group at 1 year $\left(-17.2 \mathrm{~kg} / \mathrm{m}^{2}\right)$ [17•]. The studies included had significant heterogeneity and as such outcomes with regard to resolution of co-morbidities and complications were inconsistently reported.

This heterogeneity is partly because prior to 2007 paediatric outcome data was sparse with predominantly small, retrospective, single-centre case series with different definitions and end points. There has been a real drive from the international community to quantify outcomes within this population. Three main centres have published extensively with a change in approach from retrospective to prospective collection of outcomes with results detailed below. Additionally, within the last year, the first set of prospective long-term ( $>5$ years) outcomes have been published by both the adolescent morbid obesity surgery (AMOS) and Teen-Longitudinal Assessment of Bariatric Surgery (Teen-LABS) consortiums providing an insight into the sustainability of weight loss, long-term effects on co-morbidities and the safety of bariatric surgery in childhood.

In 2012, Alqahtani et al. published the first large 3-year single-centre retrospective review of 108 paediatric patients (mean age 13.9+/-4.3 years) compared to a non-matched cohort of 114 adult patients. This demonstrated comparable percentage excess weight loss (EWL) at 2 years between the two groups (64.9 vs $69.7 \%$, respectively), despite a much higher mean BMI in the paediatric group $\left(49.6 \mathrm{vs} 32.2 \mathrm{~kg} / \mathrm{m}^{2}\right)$ [18••]. The post-operative complication rate was $5.6 \%$. Follow-up work of an increased cohort of 226 adolescent patients (mean $14.4+/-4$ ) undergoing LSG in 2014 demonstrated remission of $90.3 \%$ of co-morbidities including obstructive sleep apnoea, hypertension, diabetes and dyslipidaemia at 2 years. Importantly, although one third of all patients within this cohort were under 12 years old, all patients experienced normal growth velocity [19॰].

The Teen-LABS consortium was established in 2007 as a prospective, multi-centre observational study to measure efficacy and safety of weight loss interventions. The key to analysis was standardising management protocols, definitions and methodology. In 2014, this group published the largest series of prospectively collected data on 242 adolescent patients with a mean age of $17.1(+/-1.6)$ and BMI of 53 [20•]. Mean weight of patients decreased by $28 \%$ with gastric bypass and $26 \%$ with sleeve gastrectomy, which was sustained at 3 years. Convincing improvements were made in obesity-related comorbidities; remission of diabetes was seen in $95 \%$ of patients, with similar improvements in those with abnormal kidney function $(86 \%)$, dyslipidemia $(66 \%)$ and hypertension 
(74\%). During the 30 -day post-operative period, $8 \%$ of patients suffered a major complication and $15 \%$ defined as minor. There were no mortalities. Over 3 years follow-up, 13\% underwent one or more additional intra-abdominal procedures [21]. Since 2014, the Teen-LABS consortium has consistently published prospective results of subgroups of their cohort with regard to prevalence and resolution of obesity-associated comorbidities that will be later discussed.

The AMOS study is a Swedish multi-centre consortium producing prospective, non-randomised controlled studies, the first of which was initially published in 2012 [22•]. Outcomes of RYGB in 81 adolescents (mean age $16.5(+/-1.2)$ ) were compared with gender, age and BMI-matched controls. At 2 years follow-up, mean weight loss post-operatively was $-32 \%$ in the surgically treated adolescent group vs $+3 \%$ weight gain in the conventionally treated controls. The surgically treated adolescents were also compared to an adult cohort of gender and BMI-matched patients who achieved comparable weight loss (-31\%). A follow-up paper was published in March 2017 representing the largest 5-year follow-up study of adolescents undergoing RYGB to date [23••]. Of the adolescent surgical cohort, they had an impressive $100 \%$ follow-up rate at 5 years. Despite some limitations to the study, namely a nonrandomised control arm with non-standardised conservative treatment and a $25 \%$ cross-over to the operative arm, the authors were still able to demonstrate comparative 5-year weight loss to the 2-year results, compared with ongoing weight gain in the adolescent control group. Significant long-term improvements in co-morbidities were also seen in the surgical group with $100 \%$ resolution of type 2 diabetes and hypertension, $82 \%$ resolution of dyslipidaemia and normalisation of liver function tests in $92 \%$.

In March 2017, Inge et al. published the results of the US prospective cohort study, follow-up after bariatric surgery (FABS-5). Fifty-eight patients undergoing RYGB between 2001 and 2007 were followed up to analyse long-term outcomes with a retention rate of $81 \%$ [24••]. The mean baseline age was $17.1(+/-1.7)$ years with a mean BMI of $58.5 \mathrm{~kg} / \mathrm{m}^{2}$. Published 1-year BMI reduction was $-22.8 \mathrm{~kg} / \mathrm{m}^{2}$ and at a mean follow-up of 8 years, this weight loss was almost entirely maintained at a mean of $-16.9 \mathrm{~kg} / \mathrm{m}^{2}$ from baseline BMI at time of operation. In addition, a statistically significant ongoing decline in the prevalence of metabolic syndrome was noted with hypertension found in 19 vs $47 \%$ at operation, dyslipidaemia in 38 vs $86 \%$ and type 2 diabetes in 2 vs $16 \%$. Overall in this cohort, $63 \%$ of participants remained obese (BMI $>35 \mathrm{~kg} / \mathrm{m}^{2}$ ) at follow-up. In this study, Inge et al. further reinforced previous conclusions that there is a strong positive correlation between BMI at baseline and BMI at long-term follow-up. This continues to indicate that patients operated on earlier, at relatively lower BMIs, may achieve more successful weight loss postsurgery to normalise BMIs to non-obese levels than that seen in those with higher initial BMI.

\section{Resolution of Co-morbidities}

Increased paediatric obesity has also seen rise in the prevalence of obesity-related co-morbidities at ever younger ages, affecting almost all organ systems. It has long been recognised that obese children are at a high risk of metabolic syndrome, a constellation of glucose resistance, hypertension and hypercholesterolemia. Regression modelling from the FABS-5+ study demonstrated that there is also a significant proportional relationship between BMI and cardiometabolic risk. For every $10 \mathrm{~kg} / \mathrm{m}^{2}$ increase in BMI, there was a $34 \%$ increase in dyslipidaemia risk, $46 \%$ higher risk of hypertension and $25 \%$ rise in insulin concentration. Because the cardiovascular complications of metabolic syndrome result from cumulative years of exposure and subsequent atherosclerosis, earlier onset of obesity equates to an increased risk of premature death from cardiovascular disease. A retrospective review of 2.3 million Israeli adolescents (mean age $17.3+/-0.4$ ) by Twig et al. aimed to quantify the risk of cardiovascular death in adulthood over 43 years [25]. Hazard ratios for the obese adolescent group (BMI $>95$ th centile) as compared to the 24th centile were 4.9 for death from coronary heart disease, 2.6 for stroke and 3.5 for death from total cardiovascular causes. Adult literature has clearly demonstrated improvement in individual cardiovascular risk factors after bariatric surgery, but also a clear overall reduction of the risk of stroke, myocardial infarction and death by approximately 50\% [26]. Although there are no similar long-term studies in the adolescent population as yet, both the FABS-5+ and AMOS cohorts have demonstrated ongoing long-term regression of hypertension, dyslipidaemia and diabetes in the majority of patients. Ippisch et al. also quantified the reversibility of cardiac abnormalities in adolescents (mean age $16+/-1$ years) pre and post-bariatric surgery. Echocardiography found that predictors of future cardiovascular morbidity including the left ventricular mass, hypertrophy, diastolic function and cardiac workload all significantly improved following surgically induced weight loss in 38 adolescents over 10 months [27]. It would therefore be logical to suggest that cardiovascular morbidity and mortality should decrease in severely obese adolescents undergoing bariatric surgery.

The prevalence of type 2 diabetes mellitus (T2DM) in young people has risen significantly over the last 25 years following the rise in childhood obesity. Whilst in 1992, it accounted for $3 \%$ of all new cases of diabetes in children [28]; it currently accounts for almost 50\% [29]. The associated complications affect several organ systems with severity directly related to length of disease due to a cumulative effect. Other than a significantly increased cardiovascular risk, $\mathrm{T} 2 \mathrm{DM}$ in adults is responsible for more cases of renal failure and peripheral vascular disease leading to amputation than any other disease [30]. Adolescent type 2 diabetes also appears to be a more aggressive form with renal complications 
developing much earlier compared with type 1 diabetes; $6 \%$ of adolescents develop renal failure by age 20 years, and by 29 years of age, $2.3 \%$ have developed end-stage renal disease [31]. There also appears to be a more rapid progression to insulin requirements in adolescent T2DM when compared to type $1[32 \bullet]$.

RYGB has been shown to increase insulin sensitivity by four times in adolescents both with and without diabetes after surgery [33]. Five-year remission rates of diabetes post adolescent surgery in the AMOS and FABS-5+ cohorts are at 100 and $88 \%$, respectively, although numbers were small $(n=9$, $n=3$ ). There appears to be a greater efficacy in remission of diabetes after bariatric surgery in adolescents compared to adults, possibly due to a shorter duration of obesity and reduced severity of disease at presentation. Panunzi et al. combined data from the Swedish Obesity Subjects (SOS) trial and two randomised controlled studies in adults and found shorter diabetes duration and lower fasting glucose prior to surgery independently predicted significantly higher rates of remission [34]. This may be a further contributory factor suggesting we should be operating on adolescents earlier.

Non-alcoholic fatty liver disease (NAFLD) is strongly associated with obesity and is considered a spectrum of pathology ranging from NAFLD to non-alcoholic steatohepatitis (NASH), with a 20\% 10-year progression to cirrhosis and fibrosis. With the increasing prevalence of obesity, NASH is currently the primary cause of liver function abnormalities and chronic liver disease in children [35] and is predicted to be the most common indication for liver transplantation in the next 10 years [36]. Of 148 adolescents undergoing bariatric surgery in the Teen-LABS cohort, $59 \%$ had biopsy evidence of NAFLD with mild fibrosis seen in $18 \%$ [37]. An adult meta-analysis by Muhammadi et al. showed improvement or resolution of steatosis in over $90 \%$ of patients after bariatric surgery [38]. No such outcome data exists in children; however, the AMOS study did demonstrate a 92 and $100 \%$ resolution of alanine aminotransferase and aspartate aminotransferase to normal levels at 5-year follow-up postbariatric surgery. This does raise the question whether earlier intervention in adolescents not only prevents progression from NAFLD to NASH but also leads to normalisation of inflammation and fibrosis in patients who already have NASH at the time of surgery [39].

Obesity-associated respiratory pathologies including obstructive sleep apnoea have both immediate consequences of poor school performance and irritability alongside long-term consequences such as sustained nocturnal hypertension, left ventricular hypertrophy and increased cardiovascular and cerebrovascular morbidity and mortality [40, 41]. Adult studies have demonstrated the usefulness of bariatric surgery in improving OSA outcomes with a systematic review of 1350 patients demonstrating greater than twice the reduction in apnoea-hypopnoea index in surgical patients vs non-surgical controls [42]. Of 19 adolescents undergoing RYGB with pre-operative obstructive sleep apnoea,
Kalra et al. saw a marked decrease in severity of OSAS in all patients undergoing post-operative polysomnograms, although they were only able to follow up 53\% [43]. Further work by Amin et al. in a small, prospective study of seven adolescents undergoing surgery demonstrated a $40 \%$ reduction in apnoea score at 5 weeks compared to controls [44].

The consequences of obesity on psychosocial well-being are also not to be underestimated. Multiple studies have demonstrated significant reductions in global self-esteem and quality of life in obese youth and adolescents seeking weight loss surgery have been shown to have higher levels of depressive symptoms [45-47]. These children have also been found to have lower attainment in education and training and are more likely to not complete education, which may be partly attributable to the frequency of weight-based victimisation and stigma $[48,49]$. The AMOS collaboration demonstrated a substantial improvement in psychosocial well-being in adolescents 2 years post-gastric bypass vs controls. Symptoms of anxiety, depression, anger and disruptive behaviour were significantly reduced $(p=0.001)$ and self-esteem, self-concept and overall mood significantly improved $(p<0.001)$ [50]. However, clinically depressive symptoms were found in $19 \%$ at 2 years, with two cases of attempted suicide. The Teen-LABS consortium has also demonstrated variable mental health outcomes with surgery. Of 11 patients presenting with $\geq 1$ mental health symptom pre-surgery, remission was only found in $45 \%$ [51], although no new cases developed. These findings suggest that not all adolescents benefit psychologically from bariatric surgery, and the role of psychological screening prior to operation is essential. It may however also suggest the need to intervene earlier prior to the development of psychosocial consequences of obesity in formative adolescent years.

The Teen-LABS consortium has also shown the effects of adolescent bariatric surgery on other organ systems. A significant number of severely obese adolescents have evidence of early kidney dysfunction [52], which appears to improve after surgery in their cohort [53]. Further work by this group demonstrated significant improvements in musculoskeletal pain and mobility [54] which they previously correlated to quality of life [55]. In addition, a range of carcinomas has also been independently associated with obesity including breast, colorectal, endometrial, hepatic and pancreatic [56]. As obesity has become a phenomenon over the last 30 years, it is possible we are yet to reach the peak of this effect on cancer incidence. Although there are no current data on this from the adolescent population, adult literature shows a $30-80 \%$ reduction in cancer risk after bariatric surgery over 10 years.

\section{Side Effects and Complications}

Despite very encouraging results in both weight loss and resolution of co-morbidities comparable to adult series, surgical 
and nutritional complications need to be carefully considered when assessing the justification for adolescent bariatric surgery. A recent review of the literature by Beamish et al. compiled side effects from the four main registries of bariatric surgery in adolescents: Teen-LABS, AMOS, Saudi Arabia and the Germany Obesity Registry. Only one 30-day mortality has been reported in more than 750 cases compared to an incidence of $0.08-0.31$ in adult studies. Reporting of 'minor complications' and additional operations appeared to be higher in adolescent studies compared to adult series with 13-17\% requiring additional operations as compared to 6$7 \%$ in adult cohorts [57•].

Perioperative outcomes in 77 RYGB adolescent patients from one US centre (mean age $16.8+/-2.1$ ) reported $3 \%$ intraoperative complications and $22 \%$ perioperative complications (<30 days) [58]. These most commonly included gastro-jejunal anastomotic stricture (17\%), reoperation (13\%), anastomotic leak $(7 \%)$ and dehydration (7\%). The FABS-5+ and AMOS studies have contributed significantly to our understanding of long-term complications. Further procedures were common in both cohorts; $26 \%$ required endoscopy in the FABS-5+ group; however, the need for this was not reported in the AMOS cohort. Rates of intra-abdominal surgery were comparable between the FABS-5+ and AMOS groups at 24 and 25\%, respectively. Whilst the FABS-5+ group had a higher need for cholecystectomy ( 21 vs $11 \%$ ), only two patients $(3 \%)$ required a diagnostic laparoscopy. The need for laparoscopy for small bowel obstruction was significantly higher in the AMOS group, required in $14 \%$. This was comparable to a Swedish adult cohort, and they have since changed their practice to close the mesenteric window as a consequence of this finding.

The AMOS study showed that the cumulative hospital stay over 5 years was greater in the adolescent surgical patients than in their age-matched controls (16.1 vs. 2.8 days). Two patients did not survive at 9 and 24 months post-operatively in the FABS-5+ group; however, both cases were felt to be unrelated to the surgery. There was no mortality over 5 years in the AMOS group. This data indicates the risks of surgery need to be thoroughly considered and discussed with patients and their families when considering surgical weight-loss procedures.

In the absence of supplementation, inadequate absorption of calcium, vitamin D, iron, vitamin B1, B6, B12, A and folate can occur resulting in nutritional deficiencies [59, 60]. This can manifest clinically as peripheral neuropathy (inadequate B12), beriberi syndrome (B1 deficiency), iron deficiency anaemia and osteoporosis and osteopenia. Overall, 72\% had a variation of nutritional deficiency in the AMOS study with 'several' vitamin deficiencies identified in the FABS-5+ study, which was not further quantified. In both groups, these were predominantly mild and manageable. Anaemia was found in $46 \%$ of patients in FABS $5+$ with a higher transfusion rate ( 5 vs $2.5 \%$ ) than that found in AMOS, where only $32 \%$ were affected. The AMOS 5-year study showed that although vitamin D insufficiency was $63 \%$ in surgical group 5 years post-operatively, it was $57 \%$ in adolescent controls; however, neither study had any growth velocity data. An early study published in 1994 demonstrated only $14 \%$ of adolescents undergoing RYGB took recommended supplements [61]. This emphasises the importance of the role of the nutritionists and psychologists post-operatively to encourage compliance in this group of patients.

\section{Cost}

Though bariatric surgery clearly incurs a substantial cost at delivery, in light of the potential resolution of long-term morbidity emerging from prospective studies, it appears this cost is justified. An analysis by Teen-LABS compared the cost of adolescents with no surgery with those undergoing surgery, including rates of perioperative mortality, complications and initial morbidity over time. After 3 years, surgery led to a gain of 0.199 quality-adjusted life years (QALY) compared with no surgery at a cost of $\$ 154,684$ per QALY [62]. The trajectory showed cost decreased over time, and surgery was found to be cost effective at 5 years. Although not measured, the indirect cost must be much greater considering the improvements in psychosocial health and mobility beginning to emerge from the teen-LABS group.

\section{Conclusions}

Epidemiologic data has confirmed that paediatric obesity is increasing in prevalence and severity. Increasing studies have shown that obesity in the adolescent age group increases mortality due to a multitude of associated co-morbidities.

Multiple studies have now shown reproducible, safe and effective results of adolescent bariatric surgery with weight loss and resolution of co-morbidities equivalent to that seen in the adult population. Overall health and psychosocial wellbeing also appears to be improved. As long-term outcomes are still largely unknown and complications such as vitamin deficiency and re-operation appear to be slightly higher than that seen in the adult cohort, it is imperative that surgery should only be provided by multi-disciplinary specialist teams dedicated to the holistic care of paediatric patients to ensure safety and delivery of excellent clinical care.

\section{Compliance with Ethical Standards}

Conflict of Interest The authors declare that they have no conflict of interest.

Human and Animal Rights and Informed Consent This article does not contain any studies with human or animal subjects performed by any of the authors. 
Open Access This article is distributed under the terms of the Creative Commons Attribution 4.0 International License (http:// creativecommons.org/licenses/by/4.0/), which permits unrestricted use, distribution, and reproduction in any medium, provided you give appropriate credit to the original author(s) and the source, provide a link to the Creative Commons license, and indicate if changes were made.

\section{References}

Papers of particular interest, published recently, have been highlighted as:

- Of importance

•- Of major importance

1. Freedman DS, Mei Z, Sriniavasan SR, Berenson GS, Dietz WH. Cardiovascular risk factors and excess adiposity among overweight children and adolescents: the Bogalusa heart study. J Pediatr. 2007;150(1):12-17.e2.

2. Christou NV, Lieberman M, Sampalis F, Sampalis JS. Bariatric surgery reduces cancer risk in morbidly obese patients. Surg Obes Relat Dis. 2008;4(6):691-5. doi:10.1016/j.soard.2008.08.025.

3. Kelly AS, Barlow SE, Rao G, Inge TH, Hayman LL, Steinberger J, et al. Severe obesity in children and adolescents: identification, associated health risks, and treatment approaches: a scientific statement from the American Heart Association. Circulation. 2013;128(15):1689-712.

4. Health and Social Care Information Centre. National child measurement programme 2014-15. Healthy Lives, Healthy People; A call to action on obesity in England. 2011.

5. Ells LJ, Hancock C, Copley VR, Mead E, Dinsdale H, Kinra S et al. Prevalence of severe childhood obesity in England: 2006-2013. Arch Dis Child 2015.

6. Ogden CL, Carroll MD, Kit BK, et al. Prevalence of childhood and adult obesity in the United States 2011-2012. JAMA. 2014;311(8): 806-14.

7. $\mathrm{Ng} \mathrm{M}$, et al. Global, regional and national prevalence of overweight and obesity in children and adults during 1980-2013: a systematic analysis for the global burden of disease study 2013. Lancet. 2014;384:766-81.

8. Oude Luttikhuis H, Baur L, Jansen H, Shrewsbury VA, O'Malley C, Stolk RP, Summerbell CD. Interventions for treating obesity in children. Cochrane Database Syst Rev. 2009; 21: (1).

9. Janicke DM, Steele RG, Gayes LA, Lim CS, Clifford LM, Schneider EM, et al. Systematic review and meta-analysis of comprehensive behavioral family lifestyle interventions addressing pediatric obesity. J Pediatr Psychol. 2014;39(8):809-25.

10. Must A, Jacques PF, Dallal GE, et al. Long term morbidity and mortality of overweight adolescents. A follow-up of the Harvard growth study of 1922 to 1935. N Engl J Med. 1992;327(19):1350.

11. Berrington GA, Hartge P, Cerhan JR, et al. Body-mass index and mortality among 1.46 million white adults. N Engl J Med. 2010;363:2211-9.

12. Aikenhead A, Lobstein T, Knai C. Review of current guidelines on adolescent bariatric surgery. Clin Obes. 2011;1:3-11.

13. National Institute for Health and Clinical Excellence. CG189 obesity: identification, assessment and management. Section 1.10 [https://www.nice.org.uk/guidance/cg189/chapter/1 Recommendations\#surgical-interventions. Accessed 31 May 2017.

14. International Pediatric Endosurgery Group (IPEG). IPEG guidelines for surgical treatment of extremely obese adolescents. J Laparoendosc Adv Surg Tech A. 2009;19(Suppl. 1):xiv-vi.
15. Michalsky M, Reichard K, Inge T, Pratt J, Lenders C. ASMBS pediatric committee best practice guidelines. Surg Obes Relat Dis. 2012;8:1-7.

16. Chang SH, Stoll CR, Song J, Varela JE, Eagon CJ, Colditz GA. The effectiveness and risks of bariatric surgery: an updated systematic review and meta-analysis, 2003-2012. JAMA Surg. 2014;149(3): 275-87.

17. Black JA, White B, Viner RM, et al. Bariatric surgery for obese children and adolescents: a systematic review and meta-analysis. Obes Rev. 2013;14(8):633-44. A meta-analysis of adolescent bariatric surgery including 637 patients highlighting mean BMI change in LSG vs LAGB and RYGB.

18.• Alqahtani A, Alamri H, Elahmedi M, et al. Laparoscopic sleeve gastrectomy in adult and pediatric obese patients: a comparative study. Surg Endosc. 2012;26(11):3094-100. A three-year singlecentre retrospective review of 108 paediatric patients demonstrating comparable excess weight loss to non-matched adult controls at 2 years follow up.

19. Alqahtani AR, Elahmedi MO, Al QA. Co-morbidity resolution in morbidly obese children undergoing sleeve gastrectomy. Surg Obes Relat Dis. 2014;10:842-50. Outcomes of 226 adolescent patients undergoing bariatric surgery shown to have normal growth velocity, despite one third of patients under 12 years old.

20. Inge TH, Zeller MH, Jenkins TM, et al. Perioperative outcomes of adolescents undergoing bariatric surgery: the teen-longitudinal assessment of bariatric surgery (teen-LABS) study. JAMA Pediatr. 2014;168(1):47-53. The largest prospective, multi-center observational study in adolescents to measure efficacy and safety of weight loss interventions. Over a three year period, mean weight of decreased by $28 \%$ with gastric bypass and $26 \%$ with sleeve gastrectomy with $13 \%$ requiring additional abdominal procedures.

21. Inge TH, Courcoulas AP, Jenkins TM, et al. Teen-LABS consortium. Weight loss and health status 3 years after bariatric surgery in adolescents. N Engl J Med. 2016;374(2):113-23.

22. Olbers T, Gronowitz E, Werling M, et al. Two year outcome of laparoscopic Roux-en-Y bypass in adolescents withsevere obesity: results from a Swedish Nationwide Study (AMOS). Int J Obes (Lond). 2012;36(11):1388-95. Swedish multi-centre, prospective, non-randomised controlled study of 81 surgically treated bariatric adolescents compared to age and gender matched controls not undergoing surgery with a $\mathbf{- 3 2 \%}$ mean weight loss in the surgical cohort at 2 years compared to $+3 \%$ in the medically managed patients.

23.• Olbers T, Beamish AJ, Gronowitz E, et al. Laparoscopic Roux-enY gastric bypass in adolescents with severe obesity (AMOS): a prospective, 5-year, Swedish nationwide study. Lancet Diabetes Endocrinol. 2017;5(3):174-83. doi:10.1016/S2213-8587(16) 30424-7. The largest 5 year follow up study of adolescents undergoing RYGB to date demonstrating comparable weight loss with that found at two years, compared to ongoing weight gain in the control group. Significant long-term improvements were also found in the surgical group co-morbidities.

24.• Inge TH, Jenkins TM, Xanthakos SA, et al. Long-term outcomes of bariatric surgery in adolescents with severe obesity (FABS-5+): a prospective follow-up analysis. Lancet Diabetes Endocrinol. 2017;5:165-73. A prospective cohort study of 58 patients undergoing RYGB at 8 years. A strong positive correlation between BMI at baseline and BMI at long-term follow up was observed indicating that patients operated on earlier, at relatively lower BMIs, may achieve more successful weight loss post-surgery to normalize BMIs to non-obese levels.

25. Twig G, Yaniv G, Levine H, et al. Body mass index in 2.3 million adolescents and cardiovascular death in adulthood. N Engl J Med. 2016;24:1116-23. 
26. Beamish AJ, Olbers T, Kelly AS, Inge TH. Cardiovascular effect of bariatric surgery. Nat Rev Cardiol. 2016;13:730-43.

27. Ippisch HM, Inge TH, Daniels SR, et al. Reversibility of cardiac abnormalities in morbidly obese adolescents. J Am Coll Cardiol. 2008;51(14):1342-8. doi:10.1016/j.jacc.2007.12.029.

28. American diabetes association. Type 2 diabetes in children and adolescents. Diabetes Care. 2000;323:381-90.

29. Dabelea D, Mayer-Davis EJ, Saydah S, et al. SEARCH for diabetes in youth study. Prevalence of type 1 and type 2 diabetes among children and adolescents from 2001 to 2009. JAMA. 2014;311: 1778-86.

30. Nathan DM. Clinical practice. Initial management of glycemia in type 2 diabetes mellitus. N Engl J Med. 2002;347:1342-9.

31. Dart AB, Sellers EA, Martens PJ, Rigatto C, Brownell MD, Dean HJ. High burden of kidney disease in youth-onset type 2 diabetes. Diabetes Care. 2012;35:1265-71.

32. Shah AS, D'Alessio D, Ford-Adams ME, Desai AP, Inge TH. Bariatric surgery: a potential treatment for type 2 diabetes in youth. Diabetes Care. 2016;39(6):934-40. doi:10.2337/dc16-0067. A comprehensive review article focusing on the increasing burden of adolescent diabetes with specific reference to the aeitiology, associated complications, and outcomes in the adolescent population

33. Inge TH, Prigeon RL, Elder DA, et al. Insulin sensitivity and $\beta$-cell function improve after gastric bypass in severely obese adolescents. J Pediatr. 2015;167(5):1042-8.e1.

34. Panunzi S, Carlsson L, De Gaetano A, et al. Determinants of diabetes remission and glycemic control after bariatric surgery. Diabetes Care. 2016;39:166-74.

35. Mencin AA, Lavine JE. Non alcoholic fatty liver iseasein children. Curr Opin Clin Nutr Metab Care. 2011;14:151-7.

36. Charlton MR, Burns JM, Pedersen RA, et al. Frequency and outcomes of liver transplantation for non-alcholic steatohepatitis in the United States. Gastroenterology. 2011;141:1249-53.

37. Xanthakos SA, Jenkins TM, Kleiner DE, et al. High prevalence of non alcoholic fatty liver disease in adolescents undergoing bariatric surgery. Gastroenterology. 2015;149:623-4.

38. Muhammadi RR, Kasturi KS, Chennareddygari S, et al. Effect of bariatric surgery on non-alcoholic fatty liver disease: systematic review and meta-analysis. Clin Gastroenterol Hepatol. 2008;6: 1396-402.

39. Holterman A, Gurria J, Tanpure S, et al. Non alcoholic fatty liver disease and bariatric surgery in adolescents. Semin Paediatr Surg. 2014;23:49-57.

40. Amin RS, Kimball TR, Bean JA, et al. Left ventricular hypertrophy and abnormal ventricular geometry in children and adolescents with obstructive sleep apnea. Am J Respir Crit Care Med. 2002;165: 1395-9.

41. Harding SM. Complications and consequences of obstructive sleep apnea. Curr Opin Pulm Med. 2000;6:485-9.

42. Ashrafian H, Toma T, Rowland SP, et al. Bariatric surgery or nonsurgical weight loss for obstructive sleep apnoea? A systematic review and comparison of meta-analyses. Obes Surg. 2015;25(7): 1239-50. doi:10.1007/s11695-014-1533-2.

43. Kalra M, Inge T, Garcia V, et al. Obstructive sleep apnea in extremely overweight adolescents undergoing bariatric surgery. Obes Res. 2005;13(7):1175-9.

44. Amin R, Simakajornboon N, Szczesniak R, Inge TH. Early improvement in obstructive sleep apnea and increase in orexin levels after bariatric surgeryin adolescents and young adults. Surg Obes Relat Dis. 2017;13(1):95-100. doi:10.1016/j.soard.2016.05.023.

45. Strauss RS. Childhood obesity and self esteem. Paediatrics 2000; 105.
46. Zeller H, Inge TH, Modi AC, et al. Severe obesity and comorbid condition impact on the weight related quality of life in the adolescent patient. J Pediatr. 2015;166(3):651-659.e4.

47. Zeller MH, Roehrig HR, Modi AC, et al. Health-related quality of life and depressive symptoms in adolescents with extreme obesity presenting for bariatric surgery. Pediatrics. 2006;117:1155-61.

48. Gortmaker SL, Must A, Perrin JM, et al. Social and economic consequences of overweight in adolescence and young adulthood. N Engl J Med. 1993;329:1008-12.

49. Janssen I, Craig WM, Boyce WF, et al. Associations between overweight and obesity bullying behaviours in school aged children. Paediatrics. 2004;113:1187-93.

50. Järvholm K, Karlsson J, Olbers T, et al. Two-year trends in psychological outcomes after gastric bypass in adolescents with severe obesity. Obesity (Silver Spring). 2015;23(10):1966-72. doi:10. 1002/oby.21188.

51. Zeller MH, Pendery EC, Reiter-Purtill J, et al. From adolescence to young adulthood: trajectories of psychosocial health following Roux-en-Y gastric bypass. Surg Obes Relat Dis. 2017; doi:10. 1016/j.soard.2017.03.008.

52. Xiao N, Jenkins TM, Nehus E, et al. Teen-LABS consortium. Kidney function in severely obese adolescents undergoing bariatric surgery. Obesity (Silver Spring). 2014;22(11):2319-25. doi:10. 1002/oby.20870.

53. Nehus EJ, Khoury JC, Inge TH, et al. Kidney outcomes three years after bariatric surgery in severely obese adolescents. Kidney Int. 2017;91(2):451-8. doi:10.1016/j.kint.2016.09.031.

54. Ryder JR, Edwards NM, Gupta R, et al. Changes in functional mobility and musculoskeletal pain after bariatric surgery in teens with severe obesity: teen-longitudinal assessment of bariatric surgery (LABS) study. JAMA Pediatr. 2016;170(9):871-7. doi:10. 1001/jamapediatrics.2016.1196.

55. Bout-Tabaku S, Michalsky MP, Jenkins TM, et al. Musculoskeletal pain, self-reported physical function, and quality of life in the teenlongitudinal assessment of bariatric surgery (teen-LABS) cohort. JAMA Pediatr. 2015;169(6):552-9. doi:10.1001/jamapediatrics. 2015.0378.

56. Bhaskaran K, Douglas I, Forbes H, et al. Body mass index and an increased risk of 22 specific cancers: a population based cohort study of 5.24 million UK adults. Lancet. 2014;385:755-65.

57. Beamish AJ, Reinehr T. Should bariatric surgery be performed in adolescents? Eur J Endocrinol. 2017;176(4):D1-D15. doi:10.1530/ EJE-16-0906. A recent review of the literature debating arguments for and against bariatric surgery in adolsecents and compiling side effects from the four main registries: TeenLABS, AMOS, Saudi Arabia and the Germany Obesity Registry.

58. Miyano G, Jenkins TM, Xanthakos SA, et al. Perioperative outcome of laparoscopic Roux-en-Y gastric bypass: a children's hospital experience. J Pediatr Surg. 2013;48:2092-8.

59. Inge TH, Courcoulas AP, Jenkins TM, et al. Weight loss and health status 3 years after bariatric surgery in adolescents. N Engl J Med. 2016;374:113-23.

60. Boylan LM, Sugerman HJ, Driskell JA. Vitamin E, vitamin B-6, vitamin B-12, and folate status of gastric bypass surgery patients. J Am Diet Assoc. 1988;88:579-85.

61. Rand CS, Macgregor AM. Adolescents having obesity surgery: a 6year follow-up. South Med J. 1994;87:1208-13.

62. Klebanoff MJ, Chhatwal J, Nudel JD, et al. Cost-effectiveness of bariatric surgery in adolescents with obesity. JAMA Surg. 2017;152(2):136-41. doi:10.1001/jamasurg.2016.3640. 